\title{
Associations Between Behavioral and Neural Correlates of Inhibitory Control and Amphetamine Reward Sensitivity
}

\author{
Jessica Weafer, ${ }^{*,}$, Stephanie M Gorka ${ }^{2}$, Donald Hedeker ${ }^{3}$, Mario Dzemidzic ${ }^{4,5}$, David A Kareken ${ }^{4,5,6,7}$, \\ K Luan Phan ${ }^{2,8,9,10}$ and Harriet de Wit' \\ 'Department of Psychiatry and Behavioral Neuroscience, University of Chicago, Chicago, IL, USA; ${ }^{2}$ Department of Psychiatry, University of Illinois at \\ Chicago, Chicago, IL, USA; ${ }^{3}$ Department of Public Health Sciences, University of Chicago, Chicago, IL, USA; ${ }^{4}$ Department of Neurology, Indiana \\ University, Indianapolis, IN, USA; ${ }^{5}$ Department of Radiology and Imaging Sciences, Indiana University, Indianapolis, IN, USA; ${ }^{6}$ Department of \\ Psychiatry, Indiana University, Indianapolis, IN, USA; 7 Stark Neurosciences Research Institute, Indiana University, Indianapolis, IN, USA; ${ }^{8}$ Department \\ of Psychology, University of Illinois at Chicago, Chicago, IL, USA.; ${ }^{9}$ Department of Anatomy \& Cell Biology, University of Illinois at Chicago, Chicago, IL, \\ USA; ${ }^{10}$ Mental Health Service Line, Jesse Brown Medical Center, Chicago, IL, USA
}

\begin{abstract}
Poor inhibitory control and sensitivity to drug reward are two significant risk factors for drug abuse. Although the two have been largely viewed as separate and independent risk factors, there is new evidence to suggest that they may be related at both the behavioral and neural level. This study examined associations between behavioral and neural correlates of inhibitory control and sensitivity to the subjective rewarding effects of amphetamine in humans. Healthy volunteers $(n=63)$ first completed the stop signal task, a behavioral measure of inhibitory control. Then they participated in four sessions in which they received amphetamine $(20 \mathrm{mg})$ and placebo in alternating order, providing self-report measures of euphoria and arousal at regular intervals. Finally, a subset of participants $(n=38)$ underwent an $\mathrm{fMRI}$ scan to assess neural correlates of inhibitory control. In the first phase of the study, participants with longer stop signal reaction time (SSRT) reported greater amphetamine-induced euphoria and stimulation than those with shorter SSRT. In the second phase, fMRI of response inhibition showed the expected activation in right prefrontal regions. Further, individuals who exhibited less activation in the right middle frontal gyrus during the inhibition task reported more euphoria during the amphetamine sessions. This study is the first to show associations between poor inhibitory control and amphetamine reward sensitivity at both behavioral and neural levels in humans. These findings extend our understanding of risk for drug abuse in individuals with poor inhibitory control and suggest novel targets for prevention efforts.

Neuropsychopharmacology (2017) 42, 1905-1913; doi:I0.1038/npp.2017.6I; published online 12 April 2017
\end{abstract}

\section{INTRODUCTION}

Poor inhibitory control and sensitivity to drug reward are two significant risk factors for drug abuse. Poor inhibition is a predictor of rapid acquisition, escalation, and dysregulation of drug self-administration in animals (Dalley et al, 2007; Belin et al, 2008; Beckwith and Czachowski, 2016), and in humans, prospective studies show that disinhibition predicts the development and escalation of substance abuse later in life (Nigg et al, 2006; Rubio et al, 2008; Fernie et al, 2013). Similarly, greater sensitivity to drug-induced euphoria and stimulation promotes continued use and predicts future development of substance use disorders (Quinn and Fromme, 2011; de Wit and Phillips, 2012; King et al, 2014). Although these have been viewed as separate and

*Correspondence: Dr J Weafer, Department of Psychiatry and Behavioral Neuroscience, MC3077, University of Chicago, 584IS. Maryland Avenue, Chicago, IL 60637, USA. Tel: +773-702-5833, Fax: +773-834-7698, E-mail: jweafer@uchicago.edu

Received 18 January 2017; revised 13 March 2017; accepted 14 March 2017; accepted article preview online 17 March 2017 independent risk factors, there is new evidence to suggest that they may be related at both the behavioral and neural level. Identifying the common mechanisms underlying inhibition and drug reward sensitivity could have important implications regarding the mechanisms underlying risk for drug abuse in individuals with poor control.

Behaviorally, poor inhibitory control is related to greater sensitivity to the rewarding effects of drugs. In preclinical models, in which drug reward sensitivity is often inferred by amount of drug consumption (Stephens et al, 2010), animals with poorer inhibitory control self-administer greater amounts of cocaine, nicotine, and alcohol (Dalley et al, 2007; Belin et al, 2008; Beckwith and Czachowski, 2016). In humans, healthy volunteers with poor inhibitory control self-report greater euphoria and drug liking after amphetamine compared to placebo (Weafer and de Wit, 2013), and similar relations have also been reported with alcohol (Leeman et al, 2014; Hendershot et al, 2015). These findings provide compelling evidence that individuals with poor inhibitory control are more sensitive to drug reward. 
The behavioral links between inhibition and reward are supported by evidence that the two are related at a neurobiological level (Jentsch et al, 2014). For example, rats with fewer dopamine D2 receptors and less electrically evoked striatal dopamine release exhibit both inhibitory deficits and greater self-administration of cocaine and nicotine (Dalley et al, 2007; Diergaarde et al, 2008). In humans, individuals with fewer striatal D2 receptors report greater positive subjective response to stimulant drugs (Volkow et al, 1999; Volkow et al, 2002) and also exhibit poorer inhibitory control and less brain activation during response inhibition (Ghahremani et al, 2012; Robertson et al, 2015). Despite these promising findings, it is not known whether the neural correlates of inhibitory control are related to subjective drug reward in humans.

The present study examined the association between inhibitory control and sensitivity to amphetamine reward in healthy volunteers. Our first aim was to replicate our previous finding of an association between behavioral correlates of inhibitory control and amphetamine reward. To measure inhibitory control, participants performed the stop signal task (Logan et al, 1997), a behavioral measure of the time required to inhibit a response (stop signal reaction time; SSRT). To measure amphetamine reward, they completed a drug challenge to assess the euphorigenic effects of amphetamine ( $v s$ placebo). We hypothesized that individuals with poorer inhibitory control (longer SSRT) would report greater subjective response to amphetamine. Our second aim was to test the novel association between amphetamine reward and neural correlates of inhibitory control. Neural responses during inhibition were assessed using fMRI while subjects performed the stop signal task. We examined brain activation during inhibition in relation to amphetamine reward. The stop signal task activates rightlateralized frontal brain regions known to be involved in inhibitory control, and less right frontal activation is associated with poorer inhibition (Aron and Poldrack, 2006; Congdon et al, 2010; Kareken et al, 2013). We hypothesized that poor inhibitory control, as evidenced by less brain activation during inhibition, would be associated with greater subjective response to amphetamine.

\section{MATERIALS AND METHODS}

\section{Design}

This study assessed associations between behavioral (Aim 1) and neural (Aim 2) correlates of inhibitory control and subjective response to amphetamine (see Supplementary Figure S1 for study timeline). For the first aim, healthy volunteers completed a behavioral measure of inhibitory control (SSRT) in a drug-free state, followed by a foursession drug challenge to assess their subjective responses to amphetamine $(20 \mathrm{mg})$ or placebo. Participants received both amphetamine and placebo twice, in alternating order, with drug administered first (amphetamine or placebo) randomly assigned. For this aim, we tested associations between SSRT and ratings of euphoria and stimulation after amphetamine. For the second aim, a subset of participants who completed the first portion of the study were imaged in a drug-free state while performing the stop signal task in the scanner. This session provided a measure of brain activation during response inhibition. Most of the participants completed the imaging session 1-3 weeks after the first phase of the study. For the second aim, we examined brain activation during response inhibition in relation to subjective euphoria and stimulation following amphetamine. All behavioral and drug-challenge sessions were conducted at the University of Chicago. The imaging session took place at the University of Illinois at Chicago.

The Institutional Review Boards of both universities approved the study, and it was carried out in accordance with the Declaration of Helsinki. All participants provided written informed consent for participation.

\section{Aim 1: Behavioral Correlates of Inhibitory Control and Amphetamine Reward}

Participants. Volunteers $(n=86)$ were recruited through online and printed advertisements. Inclusion criteria were: age 21-35, BMI 19-26, at least a high school education, and English fluency. Exclusion criteria were: past year DSM-IV diagnosis, lifetime history of substance dependence, or ADHD, serious medical conditions, night shift work, smoking $>5$ cigarettes/day, use of medications other than birth control, or pregnancy, lactation, or plans to become pregnant in the next 3 months. Females not on hormonal contraception completed drug administration sessions in the follicular phase of their menstrual cycle (White et al, 2002).

Measures. Stop signal task (Logan et al, 1997). This task provided a behavioral measure of inhibitory control. Participants were instructed to respond as quickly as possible to go signals, and to inhibit responses on trials in which a stop signal (auditory tone) occurred. The duration of the stop signal delay was adjusted to target a 50\% successful inhibition rate. The task consisted of 144 go and 48 stop trials. Task data were considered valid if the following criteria were met: inhibition rate between $40-60 \%$, go accuracy $>80 \%$, and mean go $\mathrm{RT}<800 \mathrm{~ms}$.

Addiction Research Center Inventory (ARCI; Martin et al, 1971). Participants completed the ARCI to assess subjective response to amphetamine. We analyzed the MorphineBenzedrine Group (MBG, euphoric effects) and A (amphetamine-like, stimulant effects) scales, as these represent the positive, rewarding effects of amphetamine (eg, Fischman and Foltin, 1991; de Wit and Phillips, 2012).

Procedure. Participants abstained from drugs, including alcohol, for $24 \mathrm{~h}$ prior to each session, as verified by selfreport, breath alcohol, and urine screens. Participants first attended an orientation session in which they provided informed consent and were familiarized with laboratory procedures and study protocol. They then completed the stop signal task to assess drug-free levels of inhibitory control.

Supplementary Figure S1 illustrates the time line of the four drug administration sessions, in which participants received amphetamine and placebo in alternating order. Amphetamine and placebo were administered twice each to minimize the influence of day-to-day variability (Rhodes and Hawk, 2016). The drug administration sessions took place from 09:00 to 13:00 hours, and were separated by at least 
48 h. Participants were tested individually. Participants were instructed not to eat after midnight before each session and were given a light snack. To minimize drug expectancies they were told they could receive one of the following: stimulant, sedative, or placebo. They first completed a baseline (pre-drug) ARCI measure, and had blood pressure and heart rate measured. At 09:20 hours, amphetamine (20 mg) or placebo was administered under double blind conditions. Participants completed the ARCI every $30 \mathrm{~min}$ following capsule administration. Sessions ended at 13:00 hours, after confirmation that blood pressure and heart rate had returned to baseline. Upon completion of all sessions, participants were debriefed and compensated for their time.

Data analyses. SSRT from the out-of-scanner stop signal task (calculated by subtracting the final mean stop signal delay from the mean go RT) provided the behavioral measure of inhibitory control.

Associations between SSRT and subjective response to amphetamine. Two linear mixed effects models for repeated measures (Hedeker and Gibbons, 2006) were conducted in SPSS22 to examine the degree to which out-of-scanner SSRT interacted with drug and time to predict measures of subjective response: ARCI MBG (euphoria) and ARCI A (stimulation). The models included random intercept, drug, and time effects to allow for individual differences in drug response and time trends, and to account for the correlation between repeated measurements. Drug order (amphetamine or placebo administered first), age, and sex were included as model covariates. The effects of interest were the two- and three-way interactions among SSRT, drug (amphetamine $v s$ placebo), and time (linear and quadratic trends).

\section{Aim 2: Neural Correlates of Inhibitory Control and Amphetamine Reward}

Participants. A subset of subjects $(n=41)$ took part in the fMRI portion of the study. Additional exclusion criteria were contraindications for fMRI and self-reported left-handedness.

Measures. Participants performed an adapted version of the stop signal task (Kareken et al, 2013) during BOLD fMRI to assess brain activation during response inhibition. Go signals were left- or right-pointing arrows, and the stop signal was an up-pointing arrow. Participants completed three task runs ( 80 go and 40 stop trials each).

Procedure. The fMRI session followed completion of the drug administration sessions. Participants were tested for recent drug use and performed a practice block of the stop signal task outside the scanner.

Imaging acquisition and processing. Participants were imaged using a 3T GE scanner with an 8-channel head coil array. $\mathrm{T}_{1}$-weighted high-resolution anatomical images were acquired for co-registration and normalization to the MNI coordinate system. Whole-brain functional imaging was performed with a standard $\mathrm{T} 2{ }^{*}$-sensitive echo planar imaging sequence (gradient-echo; repetition time, $2000 \mathrm{~ms}$; echo time, $22.2 \mathrm{~ms} ; 64 \times 64$ matrix; $220 \times 220 \mathrm{~mm}$ field of view; flip angle, $90^{\circ} ; 3 \mathrm{~mm}$ slice thickness with no gap, 44 axial slices).

Images were processed using SPM12 (Wellcome Trust Centre for Neuroimaging). Standard preprocessing of functional images included slice-time correction, spatial realignment to correct for head motion, coregistration to the participant's $\mathrm{T}_{1}$ image and warping to MNI space, resampling to $2 \mathrm{~mm} /$ side voxels and smoothing with an $8 \mathrm{~mm}$ FWHM isotropic Gaussian kernel. The general linear model was applied to the time series, convolved with the canonical hemodynamic response function and included a $128 \mathrm{~s}$ high-pass filter. Condition effects were modeled with event-related regressors: correct go (Go) and stop (StopInh) trials, and incorrect go and stop (StopFail) trials. Effects were estimated at each voxel and for each subject, and individual participant contrast maps for StopInh $>$ Go were created (Kareken et al, 2013). Volumes were identified as motion outliers based on image intensity difference (dvars) or framewise displacement ( $\mathrm{fd}$; $>0.5 \mathrm{~mm}$ ) using FSL's motion outlier tool (Power et al, 2012). Six head motion parameters from the SPM realignment and FSL-tagged motion outlier files were included as regressors.

fMRI analyses. Brain activation during response inhibition. We conducted a second-level, random effects onesample $t$-test for StopInh $>$ Go. As our hypotheses were specific to frontal regions, statistical inferences were made based on peak voxel significance corrected for family-wise error $\left(p_{\mathrm{FWE}}<0.05\right)$ within a frontal-insular-subcortical (FIS) mask previously used for analyses with this task (Weafer et al, 2015). This $382584 \mathrm{~mm}^{3}$ (47 823 voxels) mask included the following structural regions from AAL library (TzourioMazoyer et al, 2002) available in MarsBar: medial and lateral frontal and orbital regions, bilateral precentral gyri, anterior, and middle cingulate cortex, anterior insula, and subcortical motor regions consisting of bilateral putamen, pallidum, and caudate. Analyses were restricted to this mask because it encompasses brain regions that have been previously implicated in drug reward and/or stop signal task performance (eg, Oswald et al, 2005; Courtney et al, 2012; Ghahremani et al, 2012).

Associations between brain activation during response inhibition and subjective response to amphetamine. We re-ran the second-level, random effects model with ARCI MBG and ARCI A peak change difference scores (average amphetamine peak change score minus average placebo peak change score; Mayo and de Wit, 2015) entered as covariates. Peak voxel activation $\left(p_{\mathrm{FWE}}<0.05\right)$ within the FIS mask was used as the statistical threshold.

To determine if activation within regions identified above was related not only to mean peak change difference scores but also subjective effects across time, we extracted parameter estimates/ $\beta$ weights representing BOLD response activation in arbitrary units averaged across all voxels within a $10 \mathrm{~mm}$-radius sphere surrounding the peak activation. These parameter estimates were entered as fixed effects in place of SSRT in the same linear mixed effects models described above to examine the degree to which brain activation during response inhibition interacted with drug and time trends to predict amphetamine response. 
Table I Participant Characteristics for the Aim I (behavioral) Sample $(n=63)$ and the Aim 2 (fMRI) Sample $(n=38)$

\begin{tabular}{|c|c|c|}
\hline & $\begin{array}{l}\text { Aim I: behavioral } \\
\text { sample }(n=63)\end{array}$ & $\begin{array}{c}\text { Aim 2: fMRI } \\
\text { sample }(n=38)\end{array}$ \\
\hline Gender (M:F) & $40: 23$ & $22: 16$ \\
\hline Age & $25.1(3.4)$ & $24.9(2.7)$ \\
\hline Education (years) & I5.3 (I.6) & I5.7 (I.6) \\
\hline \multicolumn{3}{|l|}{ Race } \\
\hline Caucasian & 37 & 23 \\
\hline More than one race & 9 & 7 \\
\hline African-American & 8 & 4 \\
\hline Asian & 7 & 4 \\
\hline $\begin{array}{l}\text { Hawaiian/Pacific } \\
\text { Islander }\end{array}$ & । & - \\
\hline Not reported & । & - \\
\hline \multicolumn{3}{|l|}{ Current substance use } \\
\hline Alcohol (drinks/week) & $7.3(6.7)$ & $7.0(6.0)$ \\
\hline Cigarettes (per day) & $1.0(1.5) ; n=21$ & I.I $(2.0) ; n=13$ \\
\hline Caffeine (cups/day) & $1.6(1.0) ; n=57$ & $1.7(1.1) ; n=35$ \\
\hline $\begin{array}{l}\text { Marijuana (times/ } \\
\text { month) }\end{array}$ & $16.4(17.2) ; n=30$ & $7.0(8.9) ; n=12$ \\
\hline \multicolumn{3}{|c|}{ Lifetime substance use (\% ever used) } \\
\hline Marijuana & $84.1 \%$ & $84.2 \%$ \\
\hline Hallucinogens & $38.1 \%$ & $31.6 \%$ \\
\hline Stimulants & $31.7 \%$ & $26.3 \%$ \\
\hline Opiates & $23.8 \%$ & $18.4 \%$ \\
\hline MDMA & $22.2 \%$ & $21.1 \%$ \\
\hline Sedatives & $17.5 \%$ & $7.9 \%$ \\
\hline
\end{tabular}

\section{RESULTS}

\section{Aim 1: Behavioral Correlates of Inhibitory Control and Amphetamine Reward}

Sample characteristics. A total of 86 participants completed the drug administration protocol. Of these, 63 had valid behavioral (out-of-scanner) stop signal data based on the criteria described above. Table 1 presents demographic and substance use data.

SSRT. Mean SSRT during stop signal task performance was $251.4 \mathrm{~ms} \quad(\mathrm{SD}=24.8$; range $=197.6-308.3)$. Other summary statistics were as follows: mean inhibition rate $=49.9 \%$, mean go accuracy $=98.6 \%$, and mean go $\mathrm{RT}=470.04 \mathrm{~ms}(\mathrm{SD}=97.3)$.

Associations between SSRT and subjective response to amphetamine. Table 2 presents results from the linear mixed effects models testing the degree to which SSRT interacted with drug and time to predict responses on ARCI MBG (euphoria) and ARCI A (stimulation). As expected, amphetamine increased both $\mathrm{MBG}$ and $\mathrm{A}$ ratings relative to placebo (indicated by significant drug $\times$ time interactions). Moreover, the magnitude of the amphetamine effect differed according to
Table 2 Linear Mixed Effects Models Testing Associations Between SSRT and Subjective Response to Amphetamine (Aim I)

\begin{tabular}{|c|c|c|c|c|}
\hline & Estimate & SE & $\boldsymbol{t}$ & $p$ \\
\hline \multicolumn{5}{|l|}{ ARCI MBG (Euphoria) } \\
\hline Order & 0.44 & 0.46 & 0.95 & 0.346 \\
\hline Sex & -0.19 & 0.48 & 0.41 & 0.685 \\
\hline Age & 0.01 & 0.07 & 0.13 & 0.898 \\
\hline SSRT & -0.01 & 0.02 & 0.56 & 0.578 \\
\hline Drug & -0.16 & 0.40 & 0.39 & 0.696 \\
\hline Time (linear) & -0.33 & 0.17 & 1.90 & 0.061 \\
\hline Time $^{2}$ (quadratic) & 0.04 & 0.02 & 1.92 & 0.057 \\
\hline SSRT $\times$ Drug & $<-0.01$ & 0.02 & 0.16 & 0.875 \\
\hline SSRT $\times$ Time & $<-0.01$ & 0.01 & 0.48 & 0.631 \\
\hline SSRT $\times$ Time $^{2}$ & $<0.01$ & $<0.01$ & 0.38 & 0.707 \\
\hline Drug $\times$ Time & 2.00 & 0.15 & 13.41 & $<0.001$ \\
\hline Drug $\times$ Time $^{2}$ & -0.21 & 0.02 & 10.38 & $<\mathbf{0 . 0 0 1}$ \\
\hline SSRT $\times$ Drug $\times$ Time & 0.02 & 0.01 & 3.49 & $<0.001$ \\
\hline SSRT $\times$ Drug $\times$ Time $^{2}$ & $<-0.01$ & $<0.01$ & 2.68 & 0.007 \\
\hline \multicolumn{5}{|l|}{ ARCI A (stimulation) } \\
\hline Order & 0.46 & 0.32 & 1.44 & 0.155 \\
\hline Sex & -0.41 & 0.33 & 1.24 & 0.220 \\
\hline Age & -0.01 & 0.05 & -0.25 & 0.803 \\
\hline SSRT & $<-0.01$ & 0.01 & 0.02 & 0.983 \\
\hline Drug & -0.13 & 0.26 & 0.50 & 0.619 \\
\hline Time (linear) & -0.16 & 0.07 & 2.28 & 0.023 \\
\hline Time $^{2}$ (quadratic) & 0.02 & 0.01 & 2.04 & 0.041 \\
\hline SSRT $\times$ Drug & $<0.01$ & 0.01 & 0.21 & 0.837 \\
\hline SSRT $\times$ Time & $<-0.01$ & $<0.01$ & 0.52 & 0.601 \\
\hline SSRT $\times$ Time $^{2}$ & $<0.01$ & $<0.01$ & 0.01 & 0.989 \\
\hline Drug $\times$ Time & 1.30 & 0.10 & 13.37 & $<0.001$ \\
\hline Drug $\times$ Time $^{2}$ & -0.14 & 0.01 & 10.14 & $<0.001$ \\
\hline SSRT $\times$ Drug $\times$ Time & 0.01 & $<0.01$ & 2.88 & 0.004 \\
\hline SSRT $\times$ Drug $\times$ Time $^{2}$ & $<-0.01$ & $<0.01$ & 2.28 & 0.023 \\
\hline
\end{tabular}

Abbreviation: SSRT, stop signal reaction time.

Note. Significant effects are indicated in a bold font.

individual differences in SSRT, as evidenced by the significant SSRT $\times$ drug $\times$ time interactions. To visualize the differences in drug effect according to SSRT, we plotted estimates of drug effects derived from the models at high and low levels of SSRT (1 SD above and below the mean) in Figure 1. The figure shows greater magnitude of drug effects for both $\mathrm{MBG}$ and $\mathrm{A}$ at high SSRT (1 SD above the mean; indicative of poor inhibitory control) compared to low levels of SSRT (1 SD below the mean; indicative of good inhibitory control). Thus, as hypothesized, poor inhibitory control was associated with greater amphetamine-induced euphoria and stimulation.

\section{Aim 2: Neural Correlates of Inhibitory Control and Amphetamine Reward}

Sample characteristics. In total, 41 participants completed the stop signal task during fMRI. Of these, 38 had valid in-scanner task performance (Table 1). 

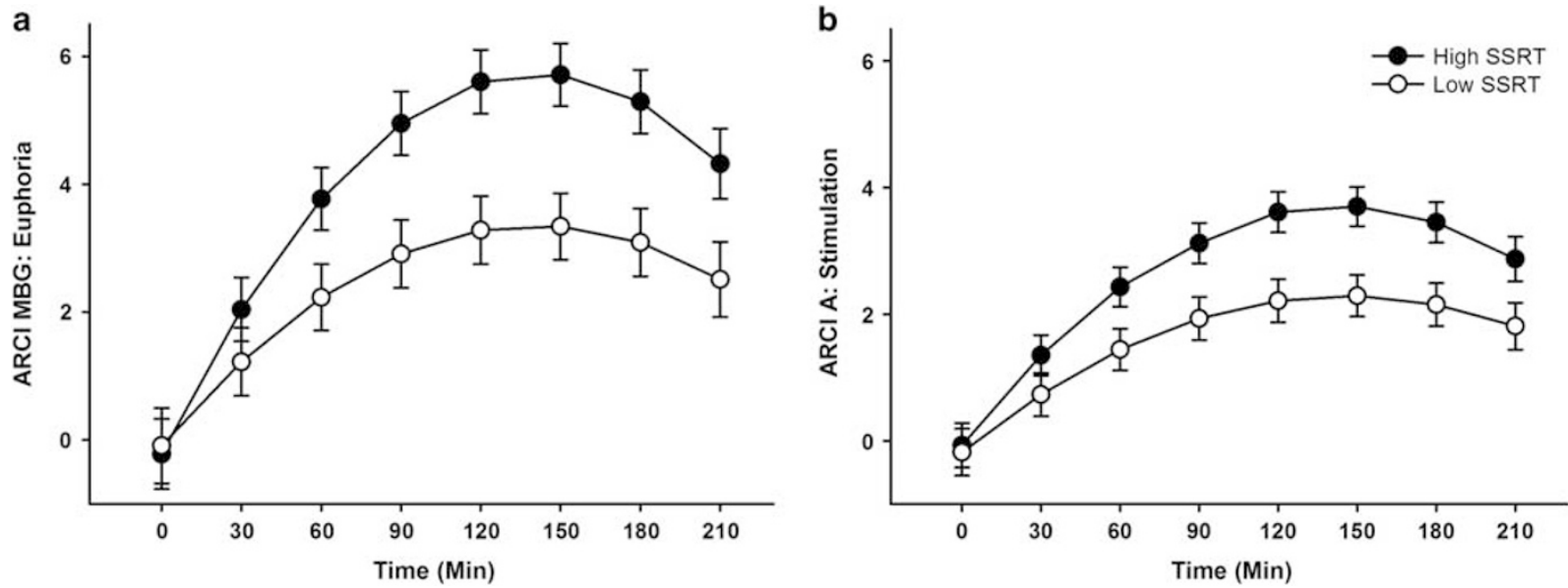

Figure I Estimates of drug effects on ARCI MBG (euphoria; a) and ARCI A (stimulation; b) derived from the linear mixed effects models testing associations between SSRT and subjective response to amphetamine (Aim I). Solid circles represent estimates of drug effects at high levels of SSRT (I SD above the mean) and open circles represent estimates of drug effects at low levels of SSRT (I SD below the mean). The inverted U reflects the typical time course of drug response to amphetamine. That is, for both high and low levels of SSRT, magnitude of drug effect increased from baseline to I20 min after drug administration. Effects peaked for about $60 \mathrm{~min}$, and then continued to decline over the session. As hypothesized, poor inhibitory control (high SSRT) was associated with greater magnitude of drug effect across time for both measures. Capped vertical lines represent SEM drug effect.

Brain activation during response inhibition. Performance validity checks confirmed that participants followed task instructions for the adapted stop signal task (mean SSRT $=312.4 \mathrm{~ms}$; mean inhibition rate $=53.6 \%$; mean go accuracy $=96.0 \%$; and mean go $\mathrm{RT}=472.5 \mathrm{~ms}$ ); SSRT obtained during fMRI was correlated with SSRT obtained during the laboratory test $(r=0.35 ; p=0.035$, one-tailed). fMRI analyses showed that the StopInh $>$ Go contrast activated regions previously reported for the stop signal task, including inferior and middle frontal gyri, precentral gyrus, insula, cingulate gyrus, and supplementary motor area (Congdon et al, 2010; Kareken et al, 2013) (Figure 2a; Supplementary Table S1).

Associations between brain activation during response inhibition and subjective response to amphetamine. StopInh $>$ Go BOLD activation was negatively correlated with peak change difference score for ARCI MBG in the right middle frontal gyrus (Figure $2 b$ ), with a significant peak voxel $\left(p_{\mathrm{FWE}}=0.042\right)$ at the $[32,48,26]$ MNI coordinate. Similarly, there was a negative correlation between StopInh $>$ Go BOLD activation and ARCI A in the right middle frontal gyrus, with a peak voxel that approached significance $\left(p_{\mathrm{FWE}}=0.074\right)$ at the $[40,14,58] \mathrm{MNI}$ coordinate. No significant positive associations were observed between brain activation during response inhibition (StopInh $>$ Go) and ARCI MBG or ARCI A. Thus, as hypothesized, less right prefrontal brain activation during response inhibition was associated with greater subjective response to amphetamine.

To further clarify the association between brain activation during response inhibition and ARCI MBG, we entered the extracted BOLD signal $(\beta$ weights, from a $10 \mathrm{~mm}$-radius sphere surrounding the significant peak voxel) in place of SSRT in the same linear mixed effects model described above. We then plotted estimates of drug effects at high (1 SD above the mean) and low (1 SD below the mean) levels of activation (Figure 2c; Table 3). Consistent with the negative correlation between right middle frontal activation and MBG scores shown in the SPM regression analysis, magnitude of drug effect was greater for lower levels of frontal activation during inhibition.

To further validate that brain activation in this region is related to successful response inhibition, we tested correlations between the extracted $\beta$ weights and SSRT obtained both during the laboratory task performance and inside the scanner. The behavioral measure of SSRT obtained during the laboratory session was significantly negatively correlated with the extracted BOLD signal $(r=-0.38, p=0.049)$, confirming that less brain activation during response inhibition was associated with longer SSRT (indicative of poor inhibition). By contrast, the in-scanner measure of SSRT was not significantly correlated with BOLD signal $(r=-0.05, p=\mathrm{NS})$.

\section{DISCUSSION}

This study yielded two important findings. First, we replicated a previous finding that individuals with poor inhibitory control experienced greater euphoria and stimulation following amphetamine (Weafer and de Wit, 2013). Second, we showed for the first time a novel association between brain activation during inhibition and amphetamine reward. Specifically, individuals with less activation in the right middle frontal gyrus during response inhibition reported greater amphetamine-induced euphoria. These findings are consistent with animal studies showing that poor inhibition predicts greater drug self-administration (Belin et al, 2008; Diergaarde et al, 2008). Importantly, they are the first to show that poor inhibitory control is associated with greater amphetamine reward at both the behavioral and neural level in humans.

The negative association between right middle frontal gyrus activation during inhibition and subjective response to amphetamine suggests a novel neurobiological link between inhibitory control and amphetamine reward. The right middle frontal gyrus has long been implicated in inhibitory 
a Brain activation during response inhibition

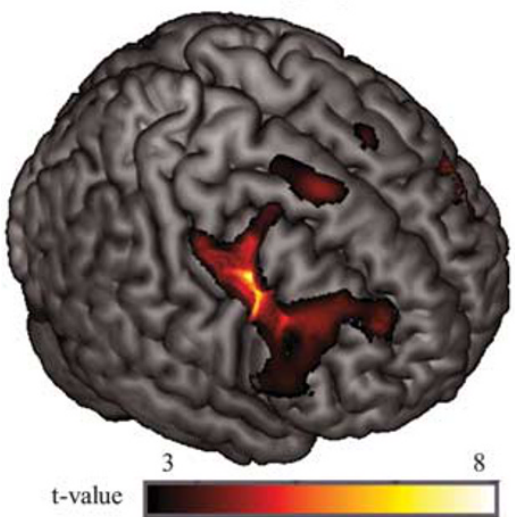

C Estimates of drug effects derived from a linear mixed effects model testing associations between peak BOLD activation (Fig $2 \mathrm{~b}$ ) and subjective response to amphetamine

b Negative association between brain activation during response inhibition and amphetamine response
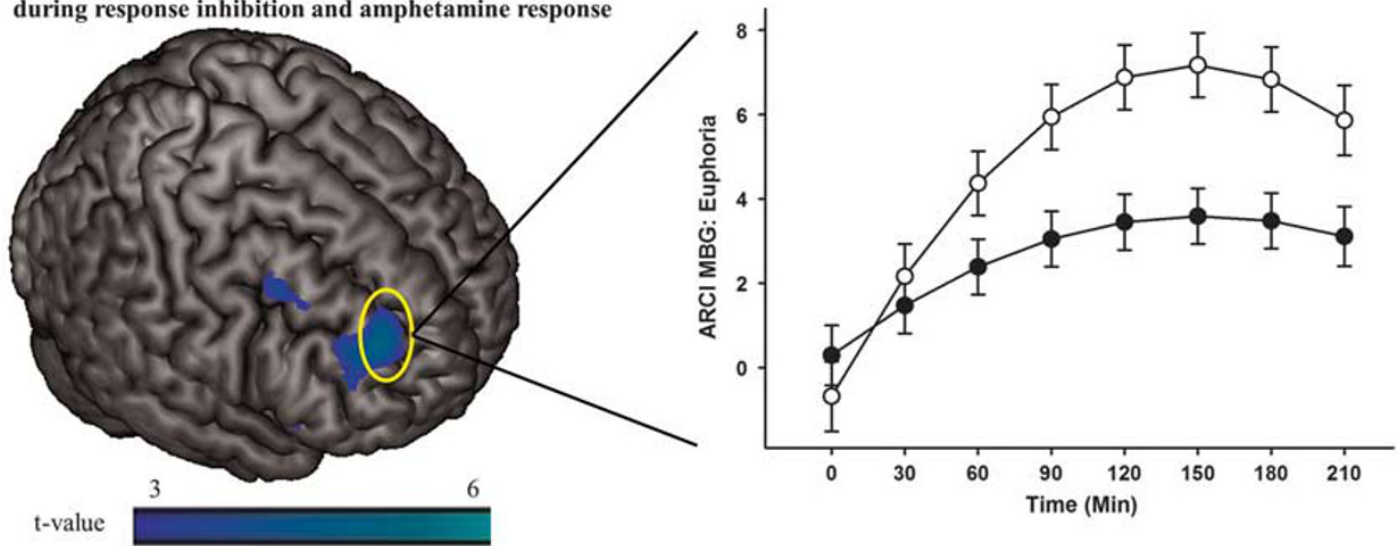

Figure 2 (a) Brain activation during successful response inhibition (Stoplnh $>$ Go) within the FIS mask. Significant ( $\left.p_{\text {FWE }}<0.05\right)$ peak activation was observed in a large right prefrontal cluster encompassing precentral, middle and inferior frontal gyri and anterior insula. Smaller middle and inferior frontal gyri and insular activations were seen in the left hemisphere (Supplementary Table SI). (b) Negative relationship between brain activation during response inhibition (Stoplnh> Go)] and ARCI MBG peak change difference scores (Aim 2). The negative relationship suggests that less prefrontal activation during inhibition is associated with greater amphetamine-induced euphoria. Peak effect at the $[32,48,26] \mathrm{MNI}$ coordinate is significant after correcting for family wise error (PFWE <0.05) within a frontal-insular-subcortical (FIS) mask. (c) Estimates of drug effects on ARCI MBG (euphoria) derived from the linear mixed effects model testing associations between brain activation during response inhibition (ie, extracted BOLD signal from a 10 mm radius spherical region centered at the $[32,48,26]$ peak in the middle frontal gyrus displayed in 2 b) and subjective response to amphetamine (Aim 2). Solid circles represent estimates of drug effects at high levels of activation (I SD above the mean) and open circles represent estimates of drug effects at low levels of activation (I SD below the mean). As hypothesized, less frontal activation during response inhibition was associated with greater magnitude of drug effect on ratings of euphoria. Capped vertical lines represent SEM drug effect.

control (Mcnab et al, 2008; Zheng et al, 2008; Ghahremani et al, 2012; Kareken et al, 2013), and is thought to influence inhibition by exerting 'top-down' executive control over lower level, sub-cortical regions, including the striatum (Bari and Robbins, 2013). As amphetamine exerts its rewarding effects via dopamine release in the striatum (Volkow et al, 1999; Drevets et al, 2001; Leyton et al, 2002), it is possible that right prefrontal regions may also exert 'top-down' control over amphetamine reward, via control of striatal dopamine. Thus, individuals with compromised prefrontal function may experience greater dopamine release, along with greater subjective reward, following amphetamine. In line with this, a recent study showed that cortical thickness in the right middle frontal gyrus was inversely related to amphetamine-induced striatal dopamine release in healthy young adults (Casey et al, 2013). In addition, preclinical studies show that manipulation of the prefrontal cortex directly modulates striatal dopamine activity (Del Arco and
Mora, 2009). Although speculative, these studies suggest that reduced right prefrontal integrity and function may increase sensitivity to amphetamine-induced reward, perhaps via compromised regulation of dopamine function.

This novel neural link between inhibitory control and drug reward sensitivity, combined with our current and previous reports of a behavioral link, provides new insight regarding the specific mechanisms underlying increased risk for drug abuse in individuals with poor inhibitory control. Recent prospective studies have shown that both behavioral and neural correlates of inhibitory control (and decreased functioning in the middle frontal gyrus in particular) predict the onset and severity of substance abuse (Rubio et al, 2008; Fernie et al, 2013; Heitzeg et al, 2015). The current findings suggest that this increased risk is due in part to greater sensitivity to the positive, euphorigenic effects of drugs. That is, once individuals with poor inhibition begin to experiment with drugs, they may experience enhanced subjective reward, 
Table 3 Linear Mixed Effects Model Testing Associations Between BOLD Activation and Subjective Response to Amphetamine (Aim 2)

\begin{tabular}{|c|c|c|c|c|}
\hline & Estimate & SE & $\mathbf{t}$ & $\mathbf{p}$ \\
\hline \multicolumn{5}{|l|}{ ARCI MBG (euphoria) } \\
\hline Order & -0.69 & 0.45 & 1.53 & 0.135 \\
\hline Sex & -0.17 & 0.45 & 0.38 & 0.708 \\
\hline Age & 0.03 & 0.08 & 0.40 & 0.694 \\
\hline BOLD activation ( $B$ weights) & 0.10 & 0.19 & 0.52 & 0.602 \\
\hline Drug & -0.19 & 0.57 & -0.34 & 0.733 \\
\hline Time (linear) & -0.17 & 0.19 & 0.89 & 0.378 \\
\hline Time $^{2}$ (quadratic) & 0.02 & 0.02 & 0.87 & 0.388 \\
\hline BOLD activation $\times$ Drug & 0.24 & 0.26 & 0.93 & 0.357 \\
\hline BOLD activation $\times$ Time & 0.06 & 0.09 & 0.66 & 0.514 \\
\hline BOLD activation $\times$ Time $^{2}$ & -0.01 & 0.01 & 0.70 & 0.487 \\
\hline Drug $\times$ Time & 2.23 & 0.18 & 12.54 & $<0.001$ \\
\hline Drug $\times$ Time $^{2}$ & -0.22 & 0.02 & -9.14 & $<0.001$ \\
\hline $\begin{array}{l}\text { BOLD } \\
\text { activation } \times \text { Drug } \times \text { Time }\end{array}$ & -0.46 & 0.08 & -5.66 & $<0.001$ \\
\hline $\begin{array}{l}\text { BOLD } \\
\text { activation } \times \text { Drug } \times \text { Time }^{2}\end{array}$ & 0.05 & 0.01 & 4.20 & $<0.001$ \\
\hline
\end{tabular}

Note: $\mathrm{BOLD}$ activation $=$ the extracted $\mathrm{BOLD}$ signal $(B$ weights, from a $10 \mathrm{~mm}$ radius sphere surrounding the significant peak voxel $[32,48,26]$ from the negative correlation between Stoplnh > Go BOLD activation and ARCI MBG.

Significant effects are indicated in a bold font.

which in turn is likely to promote continued drug-taking. Although prospective studies are needed to test this surprising association, this information could help to understand why individuals with poor inhibition are at risk for drug abuse.

The common behavioral and neural mechanisms underlying inhibition and amphetamine reward identified here suggest potential targets for drug abuse prevention in high-risk individuals. That is, to the extent that poor inhibitory control and associated dampened right prefrontal functioning produce less 'top-down' control of amphetamine reward, then enhancing function in this region would be expected to have the opposite effect and decrease drug reward sensitivity. For instance, behavioral training can be used to increase both behavioral and neural correlates of inhibitory control (Berkman et al, 2014; Liu et al, 2015; Beauchamp et al, 2016), and thus might also serve to decrease amphetamine reward sensitivity. Another means of targeting right prefrontal function is through non-invasive brain stimulation. Both transcranial magnetic stimulation and transcranial direct current stimulation over right prefrontal regions acutely modulate performance on inhibitory control tasks (Brevet-Aeby et al, 2016), and it is possible that such modulation of right prefrontal functioning could also dampen subjective drug reward.

These findings raise additional questions regarding associations between inhibition and drug reward that are beyond the scope of this study. For instance, it will be important to assess the acute effects of amphetamine on behavioral and neural correlates of inhibitory control in relation to amphetamine reward. Amphetamine typically enhances performance on the stop signal task (Perry and
Carroll, 2008), and we previously showed that individuals who displayed greater increase in inhibitory control following amphetamine also reported greater subjective response to the drug (Weafer and de Wit, 2013). However, to our knowledge, no studies to date have examined amphetamine effects on neural correlates of response inhibition, or how these effects relate to subjective response to the drug. Studies combining neuroimaging measures of amphetamine effects on both inhibitory control and reward, using fMRI and PET, will provide more direct information regarding neurobiological mechanisms that are shared by both inhibitory and reward processes.

There are some limitations of this study worth noting. First, in-scanner SSRT correlated only modestly with out-ofscanner SSRT. This was likely due to the use of a different version of the task in the scanner (ie, visual stop signals and slower presentation of go signals), as was necessary to maximize the efficiency of the event-related fMRI design. Second, in-scanner SSRT did not correlate with brain activation during inhibition. This is not surprising given that previous studies have failed to observe robust correlations between SSRT and brain activation, likely from the need for very large samples and sufficient power for voxelwise analyses (Congdon et al, 2010). It is important to note, however, that brain activation was negatively correlated with out-of-scanner SSRT, suggesting that the behavioral and brain data are tapping similar constructs. Future studies would benefit from an additional task both in and out of the scanner that probes inhibitory control, and is also predictive of drug reward, thus increasing confidence in the reliability of the observed associations. Third, we did not counterbalance the fMRI and drug administration sessions for practical and cost considerations. Although it is unlikely that drug administration influenced the fMRI session acquired 1-3 weeks later, it will be important for future studies to counterbalance fMRI sessions if possible. Fourth, we used only one dose of amphetamine, and it is conceivable that different associations would be observed with varying doses.

In sum, this study reports novel inverse associations between behavioral and neural correlates of inhibitory control and sensitivity to amphetamine reward. These findings add important information to our understanding of risk for drug abuse in those with poor control, and suggest potential behavioral and biological targets for prevention efforts. It will be important for future studies to further probe the neurobiological mechanisms underlying poor inhibitory control and sensitivity to drug reward, including the role of the dopamine system, and D2 receptor function in particular (Jentsch and Pennington, 2014). In addition, these relationships should be examined in current drug abusers, as both inhibitory control and reward networks are impaired in addiction (Spechler et al, 2016; Sutherland et al, 2012). Finally, it is important to test whether these findings generalize to other drugs of abuse.

\section{FUNDING AND DISCLOSURE}

Dr de Wit received consulting fees from Jazz Pharmaceuticals, research support in the form of a GRAND award from Pfizer, support for a research study from Insys Therapeutics, 
and a donation of a research drug from Indivior. None of these were related to the research reported here. Drs Weafer, Gorka, Hedeker, Dzemidzic, Kareken, and Phan declare no conflict of interest. The funding agencies had no involvement in the research other than financial support.

\section{ACKNOWLEDGMENTS}

This research was supported by National Institute on Drug Abuse Grants R21 DA037642 (HdW), R01 DA002812 (HdW, KLP), and National Institute on Alcohol Abuse and Alcoholism Grant K01 AA024519 (JW). DAK and MD were supported by National Institute on Alcohol Abuse and Alcoholism grant P60 AA007611.

\section{REFERENCES}

Aron AR, Poldrack RA (2006). Cortical and subcortical contributions to stop signal response inhibition: role of the subthalamic nucleus. J Neurosci 26: 2424-2433.

Bari A, Robbins TW (2013). Inhibition and impulsivity: behavioral and neural basis of response control. Prog Neurobiol 108: 44-79.

Beauchamp KG, Kahn LE, Berkman ET (2016). Does inhibitory control training transfer?: Behavioral and neural effects on an untrained emotion regulation task. Soc Cogn Affect Neurosci 11: 1374-1382.

Beckwith SW, Czachowski CL (2016). Alcohol-preferring P rats exhibit elevated motor impulsivity concomitant with operant responding and self-administration of alcohol. Alcohol Clin Exp Res 40: 1100-1110.

Belin D, Mar AC, Dalley JW, Robbins TW, Everitt BJ (2008). High impulsivity predicts the switch to compulsive cocaine-taking. Science 320: 1352-1355.

Berkman ET, Kahn LE, Merchant JS (2014). Training-induced changes in inhibitory control network activity. J Neurosci 34: 149-157.

Brevet-Aeby C, Brunelin J, Iceta S, Padovan C, Poulet E (2016). Prefrontal cortex and impulsivity: Interest of noninvasive brain stimulation. Neurosci Biobehav Rev 71: 112-134.

Casey KF, Cherkasova MV, Larcher K, Evans AC, Baker GB, Dagher A et al (2013). Individual differences in frontal cortical thickness correlate with the d-amphetamine-induced striatal dopamine response in humans. J Neurosci 33: 15285-15294.

Congdon E, Mumford JA, Cohen JR, Galvan A, Aron AR, Xue G et al (2010). Engagement of large-scale networks is related to individual differences in inhibitory control. Neuroimage 53: 653-663.

Courtney KE, Ghahremani DG, Ray LA (2012). Fronto-striatal functional connectivity during response inhibition in alcohol dependence. Addict Biol 18: 593-604.

Dalley JW, Fryer TD, Brichard L, Robinson ES, Theobald DE, Laane $\mathrm{K}$ et al (2007). Nucleus accumbens D2/3 receptors predict trait impulsivity and cocaine reinforcement. Science 315: 1267-1270.

de Wit H, Phillips TJ (2012). Do initial responses to drugs predict future use or abuse? Neurosci Biobehav Rev 36: 1565-1576.

Del Arco A, Mora F (2009). Neurotransmitters and prefrontal cortex-limbic system interactions: implications for plasticity and psychiatric disorders. J Neural Transm 116: 941-952.

Diergaarde L, Pattij T, Poortvliet I, Hogenboom F, de Vries W, Schoffelmeer AN et al (2008). Impulsive choice and impulsive action predict vulnerability to distinct stages of nicotine seeking in rats. Biol Psychiatry 63: 301-308.

Drevets WC, Gautier C, Price JC, Kupfer DJ, Kinahan PE, Grace AA et al (2001). Amphetamine-induced dopamine release in human ventral striatum correlates with euphoria. Biol Psychiatry 49: 81-96.

Fernie G, Peeters M, Gullo MJ, Christiansen P, Cole JC, Sumnall H et al (2013). Multiple behavioural impulsivity tasks predict prospective alcohol involvement in adolescents. Addiction 108: 1916-1923.

Fischman MW, Foltin RW (1991). Utility of subjective-effects measurements in assessing abuse liability of drugs in humans. $\mathrm{Br} J$ Addict 86: 1563-1570.

Ghahremani DG, Lee B, Robertson CL, Tabibnia G, Morgan AT, De Shetler $\mathrm{N}$ et al (2012). Striatal dopamine $\mathrm{D}(2) / \mathrm{D}(3)$ receptors mediate response inhibition and related activity in frontostriatal neural circuitry in humans. J Neurosci 32: 7316-7324.

Hedeker D, Gibbons RD (2006). Longitudinal Data Analysis. Wiley: Hoboken, NJ, USA.

Heitzeg MM, Cope LM, Martz ME, Hardee JE (2015). Neuroimaging risk markers for substance abuse: recent findings on inhibitory control and reward system functioning. Curr Addict Rep 2: 91-103.

Hendershot CS, Wardell JD, Strang NM, Markovich MS, Claus ED, Ramchandani VA (2015). Application of an alcohol clamp paradigm to examine inhibitory control, subjective responses, and acute tolerance in late adolescence. Exp Clin Psychopharmacol 23: 147-158.

Jentsch JD, Ashenhurst JR, Cervantes MC, Groman SM, James AS, Pennington ZT (2014). Dissecting impulsivity and its relationships to drug addictions. Ann N Y Acad Sci 1327: 1-26.

Jentsch JD, Pennington ZT (2014). Reward, interrupted: inhibitory control and its relevance to addictions. Neuropharmacology 76: 479-486.

Kareken DA, Dzemidzic M, Wetherill L, Eiler W 2nd, Oberlin BG, Harezlak J et al (2013). Family history of alcoholism interacts with alcohol to affect brain regions involved in behavioral inhibition. Psychopharmacology 228: 335-345.

King AC, McNamara PJ, Hasin DS, Cao DC (2014). Alcohol challenge responses predict future alcohol use disorder symptoms: a 6-year prospective study. Biol Psychiatry 75: 798-806.

Leeman RF, Ralevski E, Limoncelli D, Pittman B, O'Malley SS, Petrakis IL (2014). Relationships between impulsivity and subjective response in an IV ethanol paradigm. Psychopharmacology 231: 2867-2876.

Leyton M, Boileau I, Benkelfat C, Diksic M, Baker G, Dagher A (2002). Amphetamine-induced increases in extracellular dopamine, drug wanting, and novelty seeking: a PET/[C-11] raclopride study in healthy men. Neuropsychopharmacology 27: 1027-1035.

Liu Q, Zhu XY, Ziegler A, Shi JN (2015). The effects of inhibitory control training for preschoolers on reasoning ability and neural activity. Sci Rep 5: 14200.

Logan GD, Schachar RJ, Tannock R (1997). Impulsivity and inhibitory control. Psychol Sci 8: 60-64.

Martin WR, Sloan JW, Sapira JD, Jasinski DR (1971). Physiologic, subjective, and behavioral effects of amphetamine, methamphetamine, ephedrine, phenmetrazine, and methylphenidate in man. Clin Pharmacol Ther 12: 245-258.

Mayo LM, de Wit H (2015). Acquisition of responses to a methamphetamine-associated cue in healthy humans: Self-report, behavioral, and psychophysiological measures. Neuropsychopharmacology 40: 1734-1741.

Mcnab F, Leroux G, Strand F, Thorell L, Bergman S, Klingberg T (2008). Common and unique components of inhibition and working memory: an fMRI, within-subjects investigation. $\mathrm{Neu}$ ropsychologia 46: 2668-2682.

Nigg JT, Wong MM, Martel MM, Jester JM, Puttler LI, Glass JM et al (2006). Poor response inhibition as a predictor of problem drinking and illicit drug use in adolescents at risk for alcoholism and other substance use disorders. J Am Acad Child Adolesc Psychiatry 45: 468-475. 
Oswald LM, Wong DF, McCaul M, Zhou Y, Kuwabara H, Choi L et al (2005). Relationships among ventral striatal dopamine release, cortisol secretion, and subjective responses to amphetamine. Neuropsychopharmacology 30: 821-832.

Perry JL, Carroll ME (2008). The role of impulsive behavior in drug abuse. Psychopharmacology 200: 1-26.

Power JD, Barnes KA, Snyder AZ, Schlaggar BL, Petersen SE (2012). Spurious but systematic correlations in functional connectivity MRI networks arise from subject motion. Neuroimage 59: 2142-2154.

Quinn PD, Fromme K (2011). Subjective response to alcohol challenge: a quantitative review. Alcohol Clin Exp Res 35: 1759-1770.

Rhodes JD, Hawk LW (2016). Smoke and mirrors: the overnight abstinence paradigm as an index of disrupted cognitive function. Psychopharmacology 233: 1395-1404.

Robertson CL, Ishibashi K, Mandelkern MA, Brown AK, Ghahremani DG, Sabb F et al (2015). Striatal D1- and D2-type dopamine receptors are linked to motor response inhibition in human subjects. J Neurosci 35: 5990-5997.

Rubio G, Jimenez M, Rodriguez-Jimenez R, Martinez I, Avila C, Ferre $\mathrm{F}$ et al (2008). The role of behavioral impulsivity in the development of alcohol dependence: a 4-year follow-up study. Alcohol Clin Exp Res 32: 1681-1687.

Spechler PA, Chaarani B, Hudson KE, Potter A, Foxe JJ, Garavan H (2016). Response inhibition and addiction medicine: from use to abstinence. Prog Brain Res 223: 143-164.

Stephens DN, Duka T, Crombag HS, Cunningham CL, Heilig M, Crabbe JC (2010). Reward sensitivity: issues of measurement, and achieving consilience between human and animal phenotypes. Addict Biol 15: 145-168.

Sutherland MT, McHugh MJ, Pariyadath V, Stein EA (2012). Resting state functional connectivity in addiction: lessons learned and a road ahead. Neuroimage 62: 2281-2295.

Tzourio-Mazoyer N, Landeau B, Papathanassiou D, Crivello F, Etard O, Delcroix $\mathrm{N}$ et al (2002). Automated anatomical labeling of activations in SPM using a macroscopic anatomical parcellation of the MNI MRI single-subject brain. Neuroimage 15: 273-289.

Volkow ND, Wang GJ, Fowler JS, Logan J, Gatley SJ, Wong C et al (1999). Reinforcing effects of psychostimulants in humans are associated with increases in brain dopamine and occupancy of D (2) receptors. J Pharmacol Exp Ther 291: 409-415.

Volkow ND, Wang GJ, Fowler JS, Thanos P, Logan J, Gatley SJ et al (2002). Brain DA D2 receptors predict reinforcing effects of stimulants in humans: replication study. Synapse 46: 79-82.

Weafer J, de Wit H (2013). Inattention, impulsive action, and subjective response to d-amphetamine. Drug Alcohol Depend 133: 127-133.

Weafer J, Dzemidzic M, Eiler W, Oberlin BG, Wang Y, Kareken DA (2015). Associations between regional brain physiology and trait impulsivity, motor inhibition, and impaired control over drinking. Psychiatry Res 233: 81-87.

White TL, Justice AJ, de Wit H (2002). Differential subjective effects of d-amphetamine by gender, hormone levels and menstrual cycle phase. Pharmacol Biochem Behav 73: 729-741.

Zheng D, Oka T, Bokura H, Yamaguchi S (2008). The key locus of common response inhibition network for no-go and stop signals. J Cogn Neurosci 20: 1434-1442.

Supplementary Information accompanies the paper on the Neuropsychopharmacology website (http://www.nature.com/npp) 